\title{
The role of pharmacokinetic studies in drug discovery: where are we now, how did we get here and where are we going?
}

\begin{abstract}
"Although the fundamentals of what we would now recognize as pharmacokinetic theory and analysis were established by $1960 \ldots$ it took another 20 years before pharmacokinetic studies started to become integral to the drug discovery process."
\end{abstract}

\section{Keywords: DMPK • mass spectrometry imaging $\bullet$ pharmacokinetics $\bullet$ prediction}

The optimization of lead compounds into clinical candidates is a complex process involving in vitro and in vivo data, and computational models, both for exploiting structure-activity relationships, and for translating observed properties to the clinic. Characterizing exposure in animals in both efficacy and toxicity studies is a key part of this translation, but pharmacokinetic (PK) studies in animals have also become a routine component of project workflows. PK studies in animals were routinely used by drug discovery projects before the development and validation of predictive in vitro tools. It is possible that the use of PK studies today is influenced by that legacy, and strategies have not fully evolved to reflect the power of predictive tools currently available [1,2].

Although the fundamentals of what we would now recognize as pharmacokinetic theory and analysis were established by 1960 [3], it took another 20 years before PK studies started to become integral to the drug discovery process [4]. There were four keys steps that made PK optimization an achievable medicinal chemistry goal, thrusting drug metabolism and pharmacokinetics to the heart of discovery projects. The key steps in approximate chronological order were: bioanalytical: development of the thermospray interface enabling the coupling of HPLC and triple quadrupole mass spectrometers [5]; mathematical: the development of clearance concepts in pharmacokinetics, allowing AUC to be derived from dose, and rate of elimination from drug concentrations [6]; experimental: development of in vitro metabolizing systems, enabling prediction of clearance from animal and human liver preparations [7]; conceptual: the realization that drug metabolism and pharmacokinetics (DMPK) properties were driven by physicochemical and chemical properties and were therefore predictable and readily amenable to optimization [8]. With these developments not only was the need for DMPK in drug design readily recognizable, but the practical steps to deliver a cost-effective and efficient process were in place.

By definition, pharmacokinetic studies conducted in drug discovery are conducted in animals. This begs the question that merits a clear, robust answer, for ethical and scientific reasons, 'why conduct pharmacokinetic studies in animals?'

Typical pharmacokinetic studies in drug discovery are conducted in rats, less commonly in mice and dogs, at low doses $(1-3 \mathrm{mg} / \mathrm{kg})$ by the intravenous route, supplemented with oral administration, ideally utilizing a clinically relevant formulation. Tens of thousands of compounds are probably studied by the industry in this way, each year. To what end? Should compounds be selected using this data? Almost certainly not. Can the data be used in drug design? Rarely. Should the assay form part of a screening cascade to identify compounds for further testing? Only with caution.

With the number and range of DMPK assays available to the modern pharmaceu-
Peter JH Webborn

Drug Safety \& Metabolism, AstraZeneca, Alderley Park, Cheshire, UK peter.webborn@astrazeneca.com

\section{FUTURE SCIENCE part of}


tical company (>100) [9] there is plenty of scope for suboptimal strategies and wasteful/ineffective 'screening cascades'. As pharmacokinetic studies are probably the most expensive of the available DMPK assays, it is important ethically, tactically and financially to use the assays in an appropriate, effective way.

There are a wide range of valid strategies for applying DMPK optimization in drug discovery. These will depend on the nature of the organization and to some extent the disease area being targeted. The key to effective use of animal pharmacokinetic studies is to recognize that their primary value is to validate the in vitro and in silico approaches that will be used to predict pharmacokinetics in man. With this principle established, PK studies can effectively be utilized in a wide range of scenarios, and almost as importantly, a rational mechanism for management of demand for animal PK studies can be established.

Focusing on PK studies as a means of validating in vitro and in silico approaches that will be used to predict pharmacokinetics in man, avoids valuing compounds based on animal data that may not translate to man, and also adds rigor to the design and use of in vitro assays. It allows a deeper discussion of the question, 'do I, or do I not, understand the behavior of this chemical series?' For example, if the absorption of a compound in animals was found to be as predicted, based on solubility and permeability data (or predictions thereof), robust human prediction of absorption may be feasible, and in vitro data (or predictions) can be used with confidence to judge subsequent analogs. If results from the in vivo study were not as predicted, this raises fundamental questions on a number of levels. Exploration of the possible reasons for the failure of predictions is a key source of learning. In this example, a range of issues come into question, from considering a more complex intestinal biology (e.g., transporter proteins/drug metabolizing enzymes) to more complex solid-state issues, and even questions relating to the robustness of in vitro assay systems/predictions. Subsequent work on these issues could result in a fuller understanding of the compounds disposition, and introduce new challenges for making predictions of disposition in man. Conversely, the risk associated with a lack of understanding could remain, in which case this would contribute to the decision to advance compound.

The ability to make robust clearance predictions are a primary need of discovery projects, not only because of the high dependence of plasma concentration-time profiles on clearance, but because of the reliance of discovery programs on assessment of analogs based on in vitro clearance data. Again, being able demonstrate accurate prediction of clearance means that high throughput in vitro tools can be relied upon for a chemical series, and in silico tools can be used for design [10].

Managing the demand for in vivo PK studies from discovery projects is a challenge in many organizations. Often, a 'successful', 'justifiable' study is one where the compound is shown to have desirable properties (e.g., low clearance and high bioavailability), and for molecules in a new chemical series this clearly is the case. However, the temptation is to continue testing related analogs, showing maintenance of these properties. A focus on prediction prompts the question 'what have we actually learnt from this study?", and a realization that animal studies that confirm predictions that were already established in the series, add little value, and can therefore be minimized.

When it comes to candidate selection, for some, the argument, 'if I can predict the kinetics in rat and dog, I have a reasonable case to ask you to believe I can predict human kinetics' is a reasonable proposition; for others it lacks validation. This is a difficult area for novel chemistry. The argument in reverse, 'if I can't predict the kinetics in rat and dog, why should anyone think I can predict human kinetics?' may be more compelling when it comes to risk assessment for clinical progression. In either case, the importance of PK predictions to guide investment decisions is clear.

There is currently an emphasis on developing models for translation of $\mathrm{PK}$ and pharmacokineticspharmacodynamics from preclinical models to the patient, as a means of understanding the potential of novel therapies and for reducing compound attrition [11]. Quite rightly, this modeling is based on unbound drug concentrations as a readily measurable driver of efficacy. This is done based on the assumption that the unbound concentration at steady state is the same in plasma and at the target site (some nonmechanistic corrections are often applied if modelling shows that this assumption is likely to be incorrect).

Looking to the future, plasma monitoring with correction for plasma protein binding is likely to remain the standard approach for PK and pharmacokinetics-pharmacodynamics translation, and for risk assessments based on observations in toxicology studies. However, recognizing that free plasma concentrations are not always the driver of drug/metabolite effects, there is a need for a more tissue-orientated analysis. Recent advances in mass spectrometry imaging technologies, enabling simultaneous quantification of drug and metabolites in tissues, have made this an attractive approach [12]. Although, quantification to the accuracy and precision of liquid chromatography-mass spectrometry-based bioanalytical methods remains a challenge, the ability to mea- 
sure drug and biomarkers simultaneously in tissues, and more importantly in regions of tissues, is offering exciting insights into our understanding of efficacy and toxicity in preclinical models. Current techniques (e.g., matrix assisted laser desorption ionization, desorption electrospray ionization) enable detection at near cellular spatial resolutions $(10-100 \mu \mathrm{m})$, and other techniques (e.g., secondary ion mass spectrometry) may, in the future, enable a view of intracellular drug kinetics [13].

Pharmacokinetic studies are, and will remain, an important component of drug discovery programs, but to maximize value they should be highly targeted to prediction of drug efficacy and safety in man.

\section{References}

1 Jamei M, Marciniak S, Feng K, Barnett A, Tucker G, Rostami-Hodjegan A. The Simcyp population-based ADME simulator. Expert Opin. Drug Metab. Toxicol. 5(2), 211-223 (2009).

2 Kuentz M, Nick S, Parrott N, Röthlisberger D. A strategy for preclinical formulation development using GastroPlus as pharmacokinetic simulation tool and a statistical screening design applied to a dog study. Eur. J. Pharm. Sci. 27(1), 91-99 (2006).

3 Wagner JG. History of pharmacokinetics. Pharmacol. Ther. 12(3), 537-562 (1981).

4 Clark B,Smith DA. Pharmacokinetics and toxicity testing. Crit. Rev. Toxicol. 12(4), 343-385 (1984).

5 Henion JD, Maylin GA. Drug analysis by direct liquid introduction micro liquid chromatography mass spectrometry. Biomed. Mass Spectrom. 7(3), 115-121 (1980).

6 Rowland M, Benet LZ, Graham GG. Clearance concepts in pharmacokinetics. J. Pharmacokinet Biopharm. 1(2), 123-136 (1973).

7 Houston JB. Utility of in vitro drug metabolism data in predicting in vivo metabolic clearance. Biochem. Pharmacol. 47(9), 1469-1479 (1994).
Emerging mass spectrometry imaging technologies offer the potential for new insights by revealing events at the near cellular level in tissues.

\section{Financial \& competing interests disclosure}

The author has no relevant affiliations or financial involvement with any organization or entity with a financial interest in or financial conflict with the subject matter or materials discussed in the manuscript. This includes employment, consultancies, honoraria, stock ownership or options, expert testimony, grants or patents received or pending, or royalties.

No writing assistance was utilized in the production of this manuscript. Smith DA. Evolution of ADME science: where else can modeling and simulation contribute? Mol. Pharm. 10(4), 1162-1170 (2013).

9 Ballard P, Brassil P, Bui KH et al. The right compound in the right assay at the right time: an integrated discovery DMPK strategy. Drug Metab. Rev. 44(3), 224-252 (2012).

10 Riley RJ, McGinnity DF, Austin RP. A unified model for predicting human hepatic, metabolic clearance from in vitro intrinsic clearance data in hepatocytes and microsomes. Drug Metab. Dispos. 33(9), 1304-1311 (2005).

11 Peck CC. Quantitative clinical pharmacology is transforming drug regulation. J. Pharmacokinet Pharmacodyn. 37(6), 617-628 (2010).

12 Gessel MM, Norris JL, Caprioli RM. MALDI imaging mass spectrometry: spatial molecular analysis to enable a new age of discovery. J. Proteomics 107C, 71-82 (2014).

13 Ellis SR, Bruinen AL, Heeren RM. A critical evaluation of the current state-of-the-art in quantitative imaging mass spectrometry. Anal. Bioanal. Chem. 406(5), 1275-1289 (2014). 